\title{
Dynamic Modulation of Amygdala-Hippocampal Connectivity by Emotional Arousal
}

\author{
Matthias Fastenrath, ${ }^{1,2}$ David Coynel, ${ }^{1,2}$ Klara Spalek, ${ }^{1,2}$ (D) Annette Milnik, ${ }^{2,4}$ Leo Gschwind, ${ }^{1,2}$ Benno Roozendaal, ${ }^{3}$ \\ Andreas Papassotiropoulos, ${ }^{2,4,5,6}$ and Dominique J.F. de Quervain ${ }^{1,4,6}$ \\ ${ }^{1}$ Division of Cognitive Neuroscience, Department of Psychology, University of Basel, 4055 Basel, Switzerland, 2Division of Molecular Neuroscience, \\ Department of Psychology, University of Basel, 4055 Basel, Switzerland, ${ }^{3}$ Radboud University Nijmegen, Department of Cognitive Neuroscience and \\ Donders Institute for Brain, Cognition and Behaviour, 6525 EZ Nijmegen, The Netherlands, ${ }^{4}$ Psychiatric University Clinics, University of Basel, 4012 Basel, \\ Switzerland, ${ }^{5}$ Department Biozentrum, Life Sciences Training Facility, University of Basel, 4056 Basel, Switzerland, and ${ }^{6}$ Transfaculty Research Platform, \\ University of Basel, 4055 Basel, Switzerland
}

Positive and negative emotional events are better remembered than neutral events. Studies in animals suggest that this phenomenon depends on the influence of the amygdala upon the hippocampus. In humans, however, it is largely unknown how these two brain structures functionally interact and whether these interactions are similar between positive and negative information. Using dynamic causal modeling of fMRI data in 586 healthy subjects, we show that the strength of the connection from the amygdala to the hippocampus was rapidly and robustly increased during the encoding of both positive and negative pictures in relation to neutral pictures. We also observed an increase in connection strength from the hippocampus to the amygdala, albeit at a smaller scale. These findings indicate that, during encoding, emotionally arousing information leads to a robust increase in effective connectivity from the amygdala to the hippocampus, regardless of its valence.

Key words: amygdala; dynamic causal modeling; effective connectivity; emotion; hippocampus; memory

\section{Introduction}

Enhanced memory for emotional events is a well-recognized phenomenon, which has obvious adaptive value in evolutionary terms, as it is vital to remember both dangerous and favorable situations (McGaugh, 2003; de Quervain et al., 2007). Studies in animals investigating the neurobiological mechanisms underlying the enhancing effect of emotional arousal on memory consolidation have indicated that this effect depends on the influence of the amygdala upon the hippocampus in case of contextual and spatial information (McGaugh, 2002; Phelps, 2004). In addition to these well-documented effects of emotional arousal on the consolidation of long-term memory in animals, there is considerable evidence from human studies that emotional arousal regulates memory-modulatory processes already during encoding (Hamann et al., 1999; Canli et al., 2000; Hamann, 2001; Phelps, 2004; de Quervain et al., 2007; Todd et al., 2013).

\footnotetext{
Received Feb. 26, 2014; revised Aug. 29, 2014; accepted Sept. 3, 2014.

Author contributions: A.P. and D.J.F.d.Q. designed research; K.S. performed research; M.F., D.C., and A.M. analyzed data; M.F., D.C., K.S., A.M., L.G., B.R., A.P., and D.J.F.d.Q. wrote the paper.

This work was supported by Swiss National Science Foundation Sinergia Grant CRSI33_130080 to D.J.F.d.Q. and A.P. We thank Elmar Merkle, Christoph Stippich, and Oliver Bieri for granting access to the fMRI facilities of the University Hospital Basel.

The authors declare no competing financial interests.

Correspondence should be addressed to either Matthias Fastenrath or Dr. Dominique J.F. de Quervain, Division of Cognitive Neuroscience, Department of Psychology, University of Basel, Birmannsgasse 8, 4055 Basel, Switzerland, E-mail:matthias.fastenrath@unibas.ch or dominique.dequervain@unibas.ch.

DOI:10.1523/JNEUROSCI.0786-14.2014

Copyright $\odot 2014$ the authors $\quad 0270-6474 / 14 / 3413935-13 \$ 15.00 / 0$
}

Anatomical investigations have revealed that the amygdala and the hippocampus are reciprocally connected (Saunders et al., 1988; Nieuwenhuys et al., 2008). To date, there is no study assessing the bidirectional influence of emotional information on amygdala-hippocampal connectivity during encoding in healthy humans. Moreover, it is not known whether positive and negative information has similar or different effects on amygdala-hippocampal connectivity. We investigated the facilitatory effect of emotionally arousing negative and positive information during encoding by examining the strength of the reciprocal connections between the amygdala and the hippocampus. This was achieved by applying dynamic causal modeling (DCM) of fMRI data in a large population of 586 healthy human subjects who participated in a memory task with a rapid and intermingled presentation of positive, neutral, and negative pictures. DCM can be applied to test specific hypotheses about the presence, direction, and the modulators of effective connectivity between a set of predefined brain regions. DCM is described in detail previously (e.g., Friston et al., 2003; for a nontechnical introduction, see Stephan et al., 2010). In brief, neural interactions between regions are expressed by differential equations, which describe (1) how the activity in one brain region causes dynamics (i.e., rate of change) in another brain region and (2) how these interactions change under the influence of experimental conditions. In this study, we focused on the interactions between the amygdala and the hippocampus because: (1) encoding of emotional pictures leads to a coactivation of the two regions in healthy humans (Dolcos et al., 2004); (2) the hippocampus is known to play a key role in human epi- 
sodic memory (Eichenbaum, 2004); and (3) the importance of the modulatory influence of the amygdala upon the hippocampus in the enhancement of contextual and spatial memory of emotionally arousing events has been well established in animal experiments (Roozendaal and McGaugh, 2011).

\section{Materials and Methods}

Participants

We recruited healthy, young subjects in Switzerland (364 females, 222 males, mean age $=22.37$ years, $\mathrm{SD}=3.17$ years). The subjects were free of any neurological or psychiatric illness, did not take any medication at the time of the experiment (except hormonal contraceptives), and were between 18 and 35 years old. The experiments were approved by the ethics committee of the Canton of Basel. All subjects gave written informed consent before participation in the study.

\section{Procedure}

Subjects underwent four consecutive tasks: a picture encoding task, a working memory task, a free recall memory test, and a recognition task. Participants were first instructed and then trained on the picture encoding task. After training, they were positioned in the scanner and received earplugs and headphones to reduce scanner noise. Their head was fixated in the coil using small cushions, and they were told not to move their heads. Pictures were presented in the scanner using MR-compatible liquid crystal display goggles (VisualSystem; Nordic NeuroLab). Eye correction was used when necessary. The picture encoding task lasted for $\sim 20 \mathrm{~min}$. Immediately afterward, subjects performed the working memory task for $\sim 10$ additional minutes. After leaving the scanner, participants were given a surprise free recall memory test of the pictures in a separate room (no time limit was set for this task). Afterward, participants were repositioned in the scanner and performed a recognition task. To document the free recall, subjects had to write a description of the remembered pictures. A picture was scored as correctly recalled if the rater could identify the presented picture on the basis of the subject's description. Two trained investigators independently rated the descriptions for recall success (inter-rater reliability $>98 \%$ ). A third independent rater decided on pictures that were rated differently. Participants received $25 \mathrm{CHF} / \mathrm{h}$ for participation.

\section{Experimental design of the picture encoding task}

Stimuli consisted of 72 pictures that were selected from the IAPS (Lang et al., 2008), as well as from in-house standardized picture sets that allowed us to equate the pictures for visual complexity and content (e.g., human presence). Pictures received from IAPS were classified according to the IAPS valence rating; the remaining eight neutral pictures were rated based on an in-house valence rating. On the basis of normative valence scores (from 1 to 9 ), pictures were assigned to negative $(2.3 \pm 0.6)$, neutral $(5.0 \pm 0.3)$, and positive $(7.6 \pm 0.4)$ conditions, resulting in 24 pictures for each valence. Positive stimuli were initially selected to match arousal ratings of negative stimuli based on data of a pilot study in 20 subjects. Four additional pictures showing neutral objects were used to control for primacy and recency effects in memory. Two of these pictures were presented in the beginning and two at the end of the picture task. Examples of pictures are as follows: erotica, sports, and appealing animals for the positive valence; bodily injury, snake, attack scenes for the negative valence; and neutral faces, household objects, and buildings for the neutral condition. In addition, 24 scrambled pictures were used. The background of the scrambled pictures contained the color information of all pictures used in the experiment (except primacy and recency pictures), overlaid with a crystal and distortion filter (Adobe Photoshop CS3). In the foreground, a mostly transparent geometrical object (rectangle or ellipse of different sizes and orientations) was shown.

The pictures were presented for $2.5 \mathrm{~s}$ in a quasi-randomized order so that at maximum four pictures of the same category occurred consecutively. A fixation cross appeared on the screen for $500 \mathrm{~ms}$ before each picture presentation. Trials were separated by a variable intertrial period of 9-12 s (jitter) that was equally distributed for each stimulus category. During the intertrial period, participants subjectively rated the picture showing scenes according to valence (negative, neutral, positive) and arousal (large, medium, small) on a 3-point scale (self-assessment manikin) by pressing a button with a finger of their dominant hand. For scrambled pictures, participants rated form (vertical, symmetric, or horizontal) and size (large, medium, small) of the geometrical object in the foreground.

\section{MRI acquisition}

Measurements were performed on a Siemens Magnetom Verio $3 \mathrm{~T}$ whole-body MR unit equipped with a 12-channel head coil. Functional time series were acquired with a single-shot echo-planar sequence using parallel imaging (GRAPPA). We used the following acquisition parameters: TE (echo time) $=35 \mathrm{~ms}$, FOV (field of view) $=22 \mathrm{~cm}$, acquisition matrix $=80 \times 80$, interpolated to $128 \times 128$, voxel size: $2.75 \times 2.75 \times 4$ $\mathrm{mm}^{3}$, GRAPPA acceleration factor $r=2.0$. Using a midsaggital scout image, 32 contiguous axial slices placed along the anterior-posterior commissure plane covering the entire brain with a TR $=3000 \mathrm{~ms}(\alpha=$ $82^{\circ}$ ) were acquired using an ascending interleaved sequence. The first two acquisitions were discarded due to T1 saturation effects. A highresolution T1-weighted anatomical image was acquired using a magnetization prepared gradient echo sequence (MP-RAGE, TR $=2000 \mathrm{~ms}$; $\mathrm{TE}=3.37 \mathrm{~ms} ; \mathrm{TI}=1000 \mathrm{~ms}$; flip angle $=8 ; 176$ slices; $\mathrm{FOV}=256 \mathrm{~mm}$; voxel size $=1 \times 1 \times 1 \mathrm{~mm}^{3}$ ).

\section{Preprocessing and first-level analysis}

We used SPM8 (Statistical Parametric Mapping, Wellcome Trust Centre for Neuroimaging, London; http://www.fil.ion.ucl.ac.uk/spm/) implemented in MATLAB R2011b (MathWorks).

General analysis steps used to process EPI volumes. Volumes were slicetime corrected to the first slice, realigned using the "register to mean" option, and coregistered to the anatomical image by applying a normalized mutual information 3D rigid-body transformation. Successful coregistration was visually verified for each subject.

Specific analysis steps applied to determine the activity peaks at the group level. First, we smoothed the voxels of the amygdalae and hippocampi separately from each other (for details, see ROI specific smoothing). Next, we modeled event types, corresponding to the presentation of pictures and scrambled stimuli, with a canonical hemodynamic response function within the context of a GLM (for details, see GLM specification). The resulting parameter estimates were contrasted (pictures minus scrambled). Finally, we normalized the contrast images to MNI space by applying DARTEL (Ashburner, 2007) (for details, see Normalization to MNI space).

ROI specific smoothing. Slice-time corrected, realigned, and coregistered EPI volumes were smoothed in a region-specific manner to determine activity peaks at the group level. The amygdalae and hippocampi in both hemispheres were smoothed independently from other regions in the brain, thereby avoiding a mixture of data across different regions. In addition, seven other structures were also smoothed separately: thalamus, caudate, putamen, pallidum, entorhinal cortex, parahippocampal cortex, and temporal pole cortex. These additional regions were not of interest in the current study but were nonetheless included for computational expedience as they are of interest for other projects. Smoothing was applied within each region as delimited by its FreeSurfer (version 4.5, http://surfer.nmr.mgh.harvard.edu/) (Fischl et al., 2002) segmentation with an isotropic $8 \mathrm{~mm}$ full-width at half-maximum Gaussian filter using the SPM functions spm_smooth and spm_mask. The remaining brain volume was smoothed by creating a mask containing all voxels within the brain that were not part of any of the aforementioned regions. After that, the separately smoothed EPI volumes were recombined into single volumes.

GLM specification. GLMs were specified to determine the peaks at the group level and to locate the local maxima of each subject when extracting time courses. Intrinsic autocorrelations were accounted for by AR(1), and low-frequency drifts were removed via high-pass filter (time constant $128 \mathrm{~s}$ ). The effect of pictures was investigated by constructing separate regressors for the picture and the scramble condition. For each subject, evoked hemodynamic responses to event types were modeled with a $\delta$ function corresponding to presentation of each stimulus category convolved with a canonical hemodynamic response function within 
the context of a GLM. Button presses and rating scale presentation during valence and arousal ratings were modeled separately. In addition, six movement parameters from spatial realigning were included as regressors of no interest. Pictures accounting for possible primacy and recency effects were excluded from statistical analysis.

Methodological considerations. The interleaved sequence used to acquire functional time series made it a prerequisite to use slice-time correction as a first preprocessing step (Kiebel et al., 2007). Slice-timing correction methods can successfully compensate for slice-timing effects (Sladky et al., 2011). Importantly, in DCM for fMRI, the direction of causality is not identified by temporal precedence. Instead, causality is embodied by the mathematical form of the differential state equation of each region. The state equations of a given model define the system's structure (e.g., the connectivity between regions), prescribing explicitly how dynamics arise within the system (Stephan et al., 2010). Therefore, a number of DCM studies with similar TRs were previously conducted (e.g., Leff et al., 2008; Seghier and Price, 2010; Richardson et al., 2011).

\section{Anatomy: segmentation of anatomical image}

Each participant's anatomical image was automatically segmented into cortical and subcortical structures using FreeSurfer version 4.5 (Fischl et al., 2002). Labeling of the cortical gyri was based on the Desikan-Killiany atlas (Desikan et al., 2006), yielding 35 regions per hemisphere. The applied segmentation and labeling technique provides an accuracy comparable with manual labeling by experts (Fischl et al., 2002; Desikan et al., 2006).

\section{Normalization to MNI space}

Normalization was conducted by applying DARTEL, which leads to an improved registration between subjects (Ashburner, 2007; Klein et al., 2009). Normalization incorporated the following steps: (1) anatomical images of each subject were segmented using the "New Segment" procedure in SPM8; (2) the resulting gray and white matter images were used to derive a study-specific group template; (3) an affine transformation was applied to map the group template to MNI space; (4) subject-totemplate and template-to-MNI transformations were combined to create a single normalization transformation for each subject; and (5) the normalization transformation for each subject was then applied to map the following: (a) anatomical images, (b) cortical and subcortical structures retrieved from the segmentation of FreeSurfer, and (c) contrast maps from the first-level analysis to MNI space. The study-specific group template was computed from a larger population of 612 subjects, which included the 586 subjects of the present study.

\section{fMRI group statistics}

To determine the activity peaks at the group level, contrast maps in MNI space were entered in a random effects model (second-level analysis) using GLM Flex (Martinos Center and Mass General Hospital, Charlestown, MA; http://nmr.mgh.harvard.edu/harvardagingbrain/People/ AaronSchultz/GLM_Flex.html). The significance threshold was set at $p<0.05$, small volume Bonferroni-corrected for multiple comparisons within the ROIs (number of voxels, 507). We used GLM Flex as EPI sequences suffer from signal loss in the presence of magnetic field inhomogeneities that can occur close to air-tissue boundaries. The normalization procedure applied in DARTEL accurately transforms both voxels with signal and voxels with signal loss to MNI space. In SPM8, signal loss at a MNI coordinate in a functional image of only one subject leads to the exclusion of the voxel at this coordinate from the group-level analysis. Therefore, the probability of a voxel being excluded increases with sample size. GLM Flex circumvents this problem by allowing a variable number of subjects at each voxel. The minimum number of subjects per voxel was set to be 293 ( $50 \%$ of all subjects).

\section{Time course extraction from ROIs for DCM}

Smoothing can be very damaging to connectivity estimation as it leads to a mixing of BOLD time courses between regions in close proximity (Smith et al., 2011). We therefore extracted the time courses from unsmoothed and unnormalized data.

We used a combination of functional and anatomical constraints to ensure the comparability of extracted time courses across subjects. The group-level peaks in MNI space were mapped to the corresponding coordinates in the native subject space by inverting the normalization warp field of each subject (for details, see MNI space to native subject space mapping). The mapping from an atlas coordinate in MNI space to a native subject space coordinate (corresponding to the same region as identified in the atlas) was only feasible because we had used an in-house atlas based on the anatomy data of our sample (for details, see Construction of a population-average anatomical probabilistic atlas). The resulting coordinate served as a starting point to search for the subject-specific local maxima within a distance of $8 \mathrm{~mm}$, to accommodate for interindividual differences (the local maxima was defined as the effect of positive, negative, and neutral pictures vs scrambled pictures). We then extracted time courses by computing the principal eigenvariate of the data across all significant voxels ( $p<0.05$ uncorrected, minimum cluster size 3 ) within a $10 \mathrm{~mm}$ sphere and within the subject-specific anatomical mask (masks for the left and right amygdalae and hippocampi were retrieved from the FreeSurfer segmentations). The extracted time courses were adjusted to the F-contrast (i.e., effects of interest) of each subject, which effectively mean-corrects the data and removes movement artifacts based on the parameter estimates from spatial realignment. ROI time courses were then entered into the DCM models. Data from all ROIs in all subjects are a prerequisite to run DCM, as the purpose of DCM is to compare different models for an observed activation (Friston et al., 2003; Stephan et al., 2010). We therefore excluded those subjects from further analysis that did not show activation in line with the criteria defined above. This was the case for $2 \%$ of our subjects (see Results). Potential reasons for the lack of sufficiently strong activation in some subjects pertain to noise in the data or data loss but might also reflect the use of different cognitive strategies.

MNI space to native subject space mapping. The group-level peaks in MNI space were mapped to the corresponding coordinates in native subject space. Procedure: (1) the volume containing the MNI coordinate was transformed to group template space by inverting the affine transform that maps from the group template space to MNI space; (2) the volume was transformed to DARTEL imported space by inverting the subject specific DARTEL flow field using nearest-neighbor interpolation as implemented in the SPM Deformations utility; (3) coregistration of the volume to the $\mathrm{cls}^{\star}$ image in native subject space; and (4) extraction of the coordinate in native space. See the SPM 8 manual for details on DARTEL spaces (http://www.fil.ion.ucl.ac.uk/spm/).

\section{DCM: model space}

We applied bilinear, deterministic DCM with two states (version DCM10 r5236) (Marreiros et al., 2008). Each of our models consisted of two nodes, corresponding to the amygdala and the hippocampus, respectively. Based on anatomical information, we specified reciprocal intrinsic connections between the regions (Saunders et al., 1988; Nieuwenhuys et al., 2008). Extrinsic inputs to regions drive the network and quantify how brain regions respond to external stimuli. The input regressor was constructed by concatenating the onsets of all positive, negative, and neutral pictures. The input regressor entered the network by projecting either into one of the regions or to both regions, giving a total of three input possibilities to the network. Next, we constructed models that expressed different assumptions with regard to the modulation of connection strength between regions, induced by the encoding of positive, negative, or neutral pictures. The forward and the backward connections were either modulated by all three, two, one, or no condition. Modulators of the forward and backward connections were varied independently from each other. Thus, we tested all possible configurations of modulatory effects. The ensuing 64 models were then crossed with the three models representing the different input possibilities. Therefore, for each subject and hemisphere, we generated and estimated 192 models that had the same intrinsic connections but differed with regard to (1) the site of the driving input and (2) the modulatory effects.

\section{DCM: model comparison}

DCM is based on Bayesian statistics. The model evidence denotes the probability of the data given the model while adjusting for model complexity and dependencies among parameters (Stephan et al., 2009; Penny 
Table 1. Comparison between linear mixed-effects models that either do, or do not, comprise a variance covariate, related to results: parameter inference ${ }^{a}$

\begin{tabular}{|c|c|c|c|c|c|}
\hline & Akaike information criterion & Bayesian information criterion & Log-likelihood & Likelihood ratio & $p$ value \\
\hline \multicolumn{6}{|l|}{ Left hemisphere } \\
\hline Model comprising no variance covariate & -9549.83 & -9500.69 & 4782.92 & - & - \\
\hline Model comprising the variance covariate & -13828.47 & -13742.48 & 6928.24 & 4290.64 & $<4.94 \mathrm{e}-324$ \\
\hline \multicolumn{6}{|l|}{ Right hemisphere } \\
\hline Model comprising no variance covariate & -8066.88 & -8017.76 & 4041.44 & - & - \\
\hline Model comprising the variance covariate & -14439.52 & -14353.54 & 7233.76 & 6384.63 & $<4.94 \mathrm{e}-324$ \\
\hline
\end{tabular}

${ }^{a}$ Comparison between models shows that models with a covariate are superior. The smaller the Akaike information criterion or Bayesian information criterion, the better the fit.

et al., 2010). Models were compared by conducting random-effects family level inference (Stephan et al., 2009; Penny et al., 2010). The model space described above was partitioned into three families based on differences in the location of the driving input. This allowed us to test whether certain input configurations were more likely than others while controlling for the effect of modulatory parameters. The likelihood is expressed as the exceedance probability, which denotes the probability that a particular model family is more likely than any other tested model family. The exceedance probabilities are easily interpretable as they sum to one over all model families tested.

\section{DCM: parameter analysis}

We applied Bayesian model averaging (BMA) to obtain a summary measure of likely connectivity values (Penny et al., 2010). The connectivity parameters of each of the 64 models within the winning family were weighted by the posterior model probability and then averaged within each subject. Those models for which the posterior probability was very low $(<1 / 20$ of the maximum probability) were excluded from the calculations for computational expedience. Overall, the BMA weighting procedure resulted in subject-specific connectivity estimates that were independent of a particular model within the winning family while ensuring that models with a high probability contributed more than models with a lower probability.

\section{DCM: group-level inference on parameters}

The BMA modulatory parameter estimates of each subject were entered into a linear mixed-effects analysis using R's (http://www.r-project.org) lme function in the nlme package (R Core Team, 2012). By default, two-state DCM BMA parameter estimates are in log-space, and these values were used to compute statistics. As fixed effects, we entered direction (Amy to Hip, Hip to Amy), stimulus category (positive, neutral, negative), and the interaction term between direction and stimulus category. Subjects were entered as random effect. The models were estimated using restricted maximum likelihood. Visual inspection of residual plots revealed deviations from homoscedasticity. To avoid a violation of statistical assumptions, we specified an exponential function of the fitted values as a variance covariate for the factors valence and direction using the varFunc function. Both visual inspection of residual plots and likelihood ratio tests between the models assuming either heteroscedasticity or homoscedasticity showed that the models with the variance covariate were superior (Table 1; Fig. 1). We checked for violation of normality by plotting the residuals of the mixed-effects models (Fig. 2). $p$ values were obtained with $\chi^{2}$ tests using the ANOVA function implemented in the car package. Two-sided paired $t$ tests were used for focused tests between modulators of connection strength.

\section{Behavioral data}

Two-sided paired $t$ tests were applied to test for differences between the number of pictures recalled per stimulus category. Picture-specific arousal ratings were averaged across all pictures of a stimulus category within each subject and then entered in two-sided paired $t$ tests to assess differences in the arousal per stimulus category. Furthermore, we computed a Pearson linear correlation coefficient to assess whether the subject-specific average arousal rating is associated with the subject-specific number of recalled pictures. $p$ values for the correlation were obtained using two-sided tests. Data from picture recall and arousal ratings were plotted and were found to be approximately normally distributed.
Construction of a population-average anatomical probabilistic atlas

Segmentations of cortical and subcortical structures retrieved from FreeSurfer were used to build a population-average probabilistic anatomical atlas. Individual segmented anatomical images were then normalized to the study-specific anatomical template space using the subject's previously computed warp field, and affine-registered to the MNI space. Nearest-neighbor interpolation was applied to preserve labeling of the different structures. The normalized segmentations were finally averaged across subjects to create a population-average probabilistic atlas. Each voxel of the template could consequently be assigned a probability of belonging to a given anatomical structure, based on the individual information from the 612 subjects. The voxels within the contours in Figure 5 could be assigned to either the amygdala or the hippocampus in at least $75 \%$ of the subjects.

\section{Exclusion criteria}

A total of 26 subjects were excluded after the preprocessing of the data and were not part of the DCM analysis. Reasons for exclusion were defined as follows: missing or corrupted functional data; subjects took part in a similar experiment and were therefore informed about the surprise free recall test; insufficient quality of anatomical image; failed coregistration; subjects that did not recall a single picture within one valence category.

\section{Results}

\section{Behavioral data: enhanced memory recall for emotionally arousing pictures}

The behavioral results across all subjects $(N=586)$ confirm that emotionally arousing pictures were recalled more often than neutral pictures (Fig. 3). Memory was assessed in a surprise free recall memory test 10 min after encoding, where 24 pictures for each stimulus category had been presented. Neutral pictures were least often recalled (neutral: $6.85 \pm 0.12$, mean \pm SD; positive vs neutral, $p=1.78 \mathrm{e}-170$; negative vs neutral, $p=1.40 \mathrm{e}-135)$. Positive pictures were slightly more often remembered than negative pictures (positive: $12.02 \pm 0.14$; negative: $11.15 \pm 0.13$; positive vs negative, $p=1.16 \mathrm{e}-10)$. Furthermore, on a 3 -point scale $(2=$ large, $1=$ medium, $0=$ small), neutral pictures were rated as least arousing (neutral: $0.38 \pm 0.01$; negative vs neutral, $p=1.96 \mathrm{e}-$ 311; positive vs neutral, $p=2.04 \mathrm{e}-184$; Fig. 4 ). Negative pictures were rated as more arousing than positive pictures (negative: $1.36 \pm 0.01$; positive: $0.92 \pm 0.02$; negative vs positive, $p=9.10 \mathrm{e}$ 124). $p$ values denote the results from two-sided paired $t$ tests. These results show that emotionally arousing pictures are better remembered than less arousing neutral pictures. We did not observe significant associations between interindividual differences in mean arousal ratings per stimulus category and interindividual differences in the number of recalled pictures per stimulus category (i.e., subjects who generally rated pictures as more arousing did not have a better memory performance than subjects who generally rated pictures as less arousing) (positive: $r=0.1, p=$ 0.02; neutral: $r=0.09, p=0.03$; negative: $r=0.01, p=0.75$; Bonferroni multiple comparison corrected significance threshold for three tests, $p=0.017$ ). 

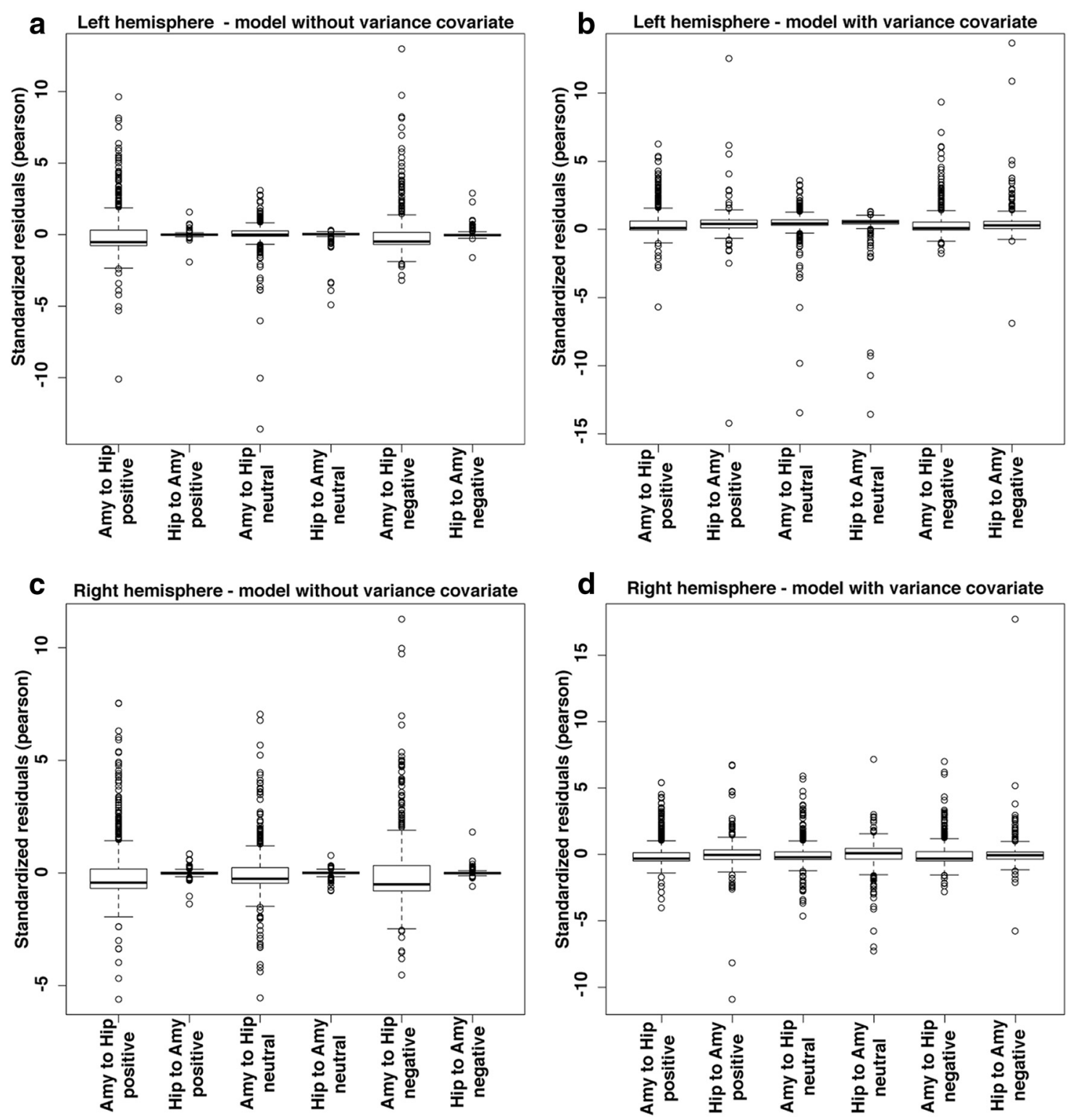

Figure 1. Box plots representing the variance of residuals per factor level of the linear mixed-effects models. Results obtained in the left hemisphere $(\boldsymbol{a}, \boldsymbol{b}, N=574)$ and right hemisphere $(\boldsymbol{c}, \boldsymbol{d}$, $N=573)$. ANOVA of modulator values is based on the models comprising the variance covariate $(\boldsymbol{b}, \boldsymbol{d})$. Models without a variance covariate $(\boldsymbol{a}, \boldsymbol{c})$ were rejected. Pearson standardized residuals are shown for each level of the two modeled factors: stimulus category (positive, neutral, negative) and direction of influence (Amy to Hip, Hip to Amy). Boxes represent the interquartile range between the first and third quartiles, and the horizontal lines within boxes indicate the median. Horizontal bars outside the boxes represent the lowest and highest values within $1.5 \times$ interquartile range from the first and third quartiles, respectively. Circles represent outside values. Amy, Amygdala; Hip, hippocampus.

\section{Peak activity within the amygdala and the hippocampus}

First, we determined the locations of the activity peaks within the amygdala and the hippocampus, as measured by fMRI recordings during encoding. The local maxima for the effect of pictures (positive + negative + neutral vs scrambled pictures) were located at MNI $-19.25,-8.25,-16$ in the left amygdala $(t=$ $38.86)$ and at MNI $-22,-16.5,-16$ in the left hippocampus ( $t=$ 49.08; Fig. 5). Local maxima in the right hemisphere were located at MNI $22,-8.25,-16$ in the amygdala $(t=39.81)$ and at MNI $22,-16.5,-16$ in the hippocampus $(t=47.97)$. All $t$ values were significant $(p<0.05$, small volume Bonferroni-corrected, threshold $t=3.9$ ). The coordinates of the four local maxima corresponded to the location of the respective anatomical structure in all subjects (regional probability in accordance to inhouse atlas: 100\%; see Materials and Methods). For activation peaks outside of amygdala and hippocampus, see Table 2.

\section{Time course extraction from ROIs in the amygdala and} the hippocampus

Before the actual modeling of connectivity within the DCM framework, time courses (i.e., the BOLD responses measured during the course of the experiment) were extracted from the locations of the activity peaks. Within the left hemisphere, time courses were successfully extracted from 574 of a total of 586 subjects (98\%). Twelve subjects ( 8 females, 4 males) were excluded from further analysis, as they did not show robust task-dependent activation in the amygdala in accordance with our criteria (see Time course extraction from ROIs for DCM). In addition, the hippocampus was also not robustly activated in one of these subjects. Within the right hemisphere, time courses were successfully extracted from 573 subjects (98\%). Twelve subjects ( 7 females, 5 males) did not show robust activation within the amygdala, and one additional male subject did not show robust activation in the hippocampus. 

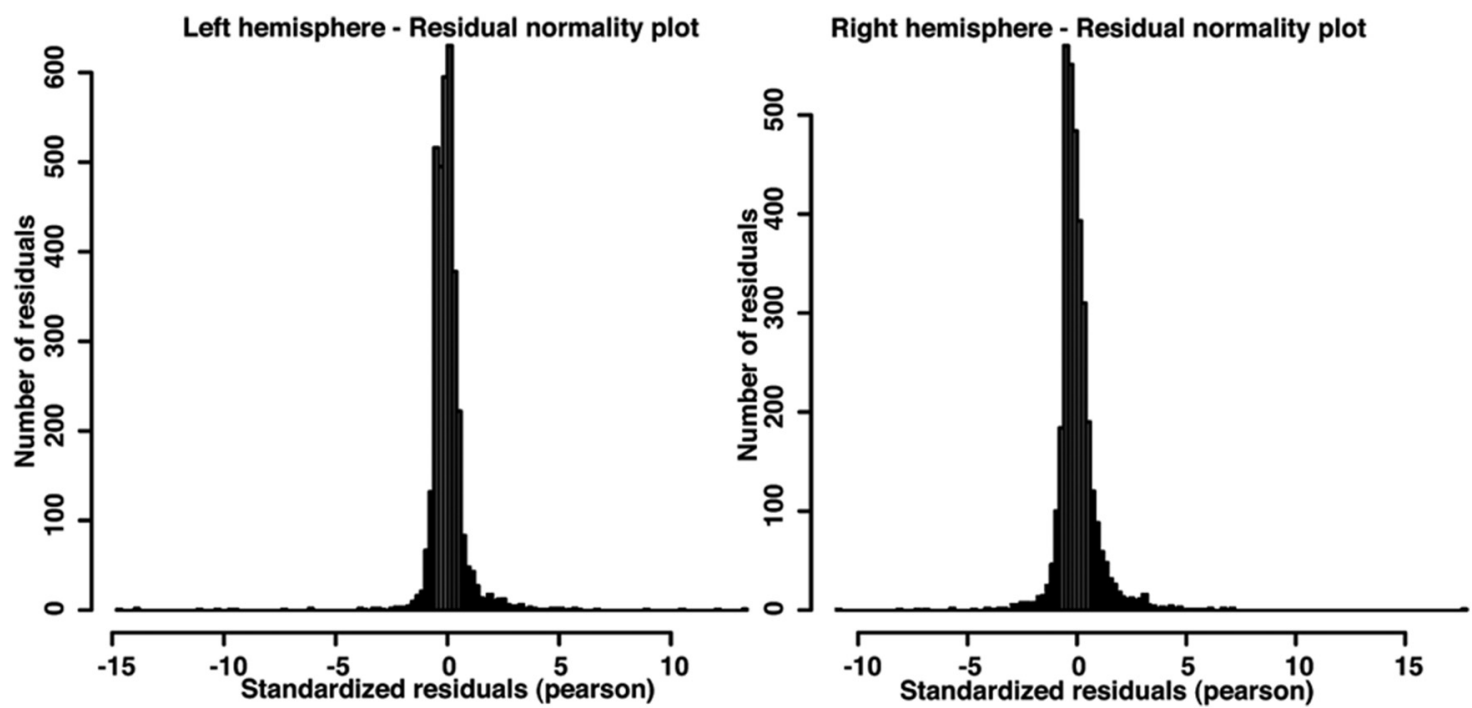

Figure 2. Distribution of model residuals. Plots representing the distribution of model residuals in the left hemisphere $(N=574)$ and right hemisphere $(N=573)$. The plots refer to the linear mixed-effects models comprising a variance covariate. Residuals of all factor levels are shown jointly.

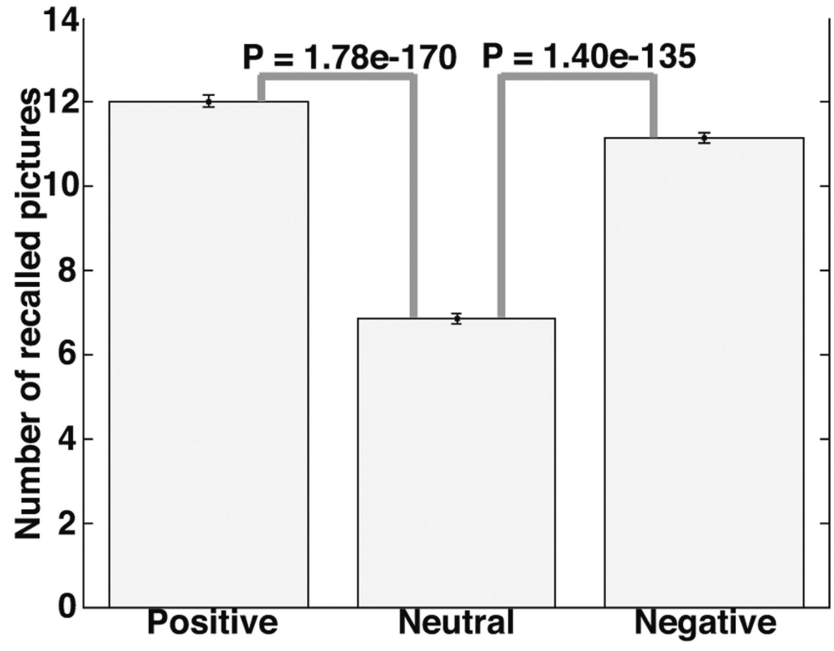

Figure 3. Average number of recalled pictures per stimulus category. Twenty-four pictures for each stimulus category (positive, neutral, negative) were presented during the encoding phase. Memory was assessed in a surprise free recall memory test 10 min after encoding. $p$ values indicate the results from two-sided paired $t$ tests, which were conducted to examine differences in the number of recalled pictures per stimulus category $(N=586)$. Error bars indicate the SEM.

DCM model comparison: determining the most likely site of input

Within the DCM framework, different competing models can be specified and formally compared by using a Bayesian model selection procedure, which identifies the most plausible family of models given the extracted time courses. In the absence of $a$ priori information about the exact site of input to the network, we compared three different model families, each representing one of the three possible input configurations: (1) input enters only the amygdala; (2) input enters only the hippocampus; and (3) input enters both the amygdala and the hippocampus. Comparison between the three model families clearly indicated that those models where the input enters only the amygdala are most likely (left hemisphere family exceedance probability $=0.9998$, right hemisphere family exceedance probability $=0.8146$; Fig. 6 ).

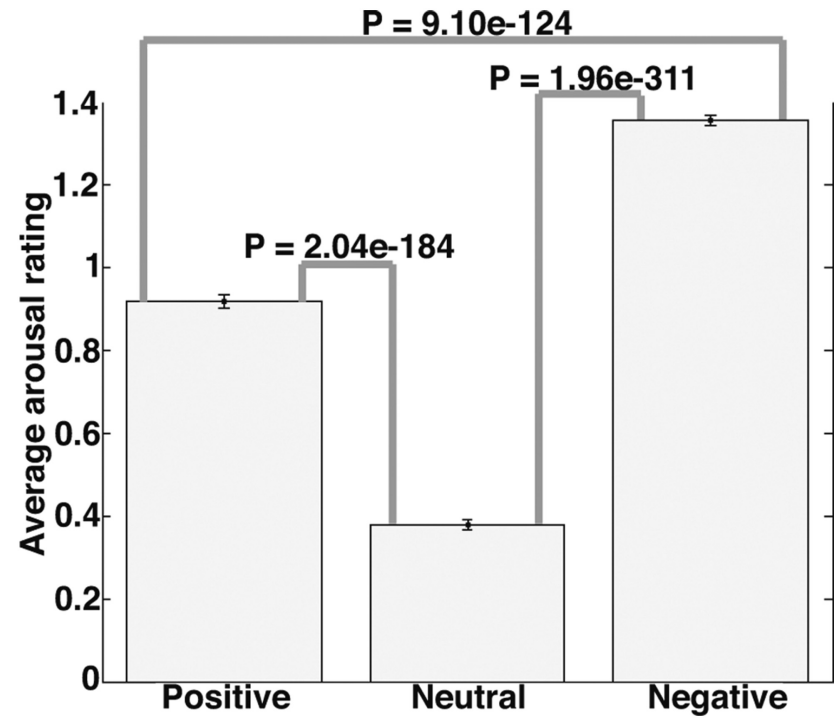

Figure 4. Average arousal rating per valence category. Participants subjectively rated each picture with regard to arousal $(2=$ large; $1=$ medium; $0=$ small $)$ on a 3 -point scale. $p$ values indicate the results from two-sided paired $t$ tests, which were conducted to examine differences in the arousal rating per stimulus category $(N=586)$. Error bars indicate the SEM.

Parameter inference: the neuromodulatory effect of emotions on connection strength

Modulators of effective connectivity describe whether the strength of the connection, (i.e., the influence that one region exerts upon another) increases or decreases under the influence of experimental manipulations. Modulator estimates are based on the complete set of positive, neutral, and negative pictures presented in the encoding task.

The influence of the amygdala upon the hippocampus was particularly strong during the encoding of both positive and negative pictures (Figs. 7 and 8), which is reflected in the results of an ANOVA with the factors direction and stimulus category (main effect of direction: left hemisphere, $\chi^{2}=132.31, p=1.28 \mathrm{e}-30$; right hemisphere, $\chi^{2}=298.87, p=5.82 \mathrm{e}-67$; main effect of stimulus category: left hemisphere, $\chi^{2}=91.49, p=1.36 \mathrm{e}-20$; right hemisphere, $\chi^{2}=74.19, p=7.74 \mathrm{e}-17$; interaction between 

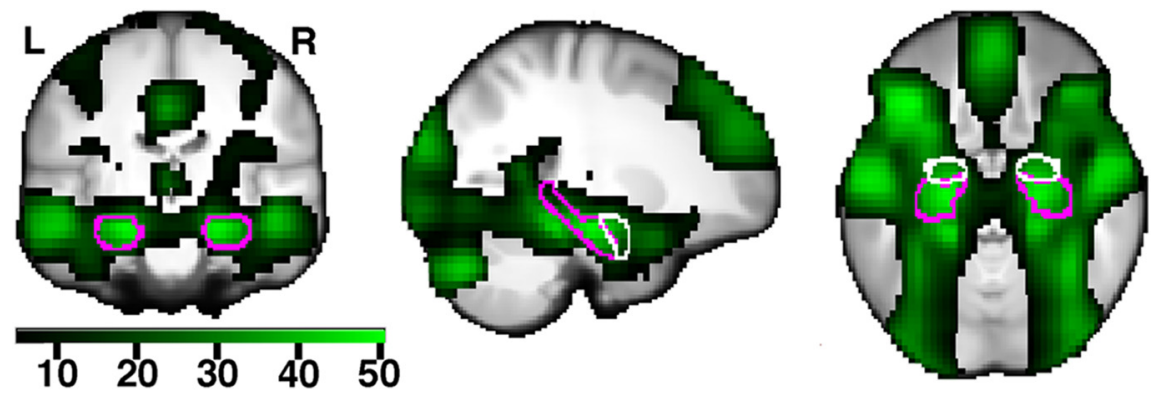

Figure 5. Brain activity related to picture encoding (pictures of all valence categories compared with scrambled pictures). Random-effects analysis across all 586 subjects ( $p<0.05$, whole-brain Bonferroni correction), illustrated using color-coded $t$ values. The image is centered at the activity peak within the left hippocampus (MNI space: $-22,-17,-16$ ). The white and violet contours comprise voxels belonging to the amygdala and to the hippocampus, respectively. Contours are retrieved from an in-house atlas (see Materials and Methods). Activations are overlaid on coronal (left), sagittal (middle), and axial sections of the study-specific group template. L, Left hemisphere; R, right hemisphere.

direction and stimulus category: left hemisphere, $\chi^{2}=83.31, p=$ 8.11e-19; right hemisphere, $\left.\chi^{2}=15.35, p=4.64 \mathrm{e}-04\right)$.

We conducted two-sided paired post hoc $t$ tests between modulators within each connection to obtain more focused results (Bonferroni multiple comparison corrected significance threshold for six tests: $p=0.008$ ). Within the connection from the amygdala to the hippocampus, these analyses showed that modulators of positive and negative pictures were not significantly different from each other (left hemisphere, $p=0.43$; right hemisphere, $p=0.34$ ), whereas neutral pictures had a comparatively smaller effect on connection strength (left hemisphere, positive vs neutral, $p=4.11 \mathrm{e}-14$; negative vs neutral, $p=3.48 \mathrm{e}-14$; right hemisphere, positive vs neutral, $p=0.0015$; negative vs neutral, $p=6.36 \mathrm{e}-05)$. Within the connection from the hippocampus to the amygdala, positive, neutral, and negative pictures had a differential effect in the left hemisphere (positive vs neutral, $p=$ 3.32e-06; negative vs neutral, $p=4.48 \mathrm{e}-10$; negative vs positive, $p=5.32 \mathrm{e}-05$ ), whereas modulators of positive and negative pictures were not significantly different in the right hemisphere (positive vs neutral, $p=8.95 \mathrm{e}-06$; negative vs neutral, $p=1.52 \mathrm{e}$ 12 ; positive vs negative, $p=0.07$ ).

We investigated whether our findings depend substantially on the sex of our subjects. We did not find a significant main effect of sex (left hemisphere, $p=0.18$; right hemisphere, $p=0.93$ ), when sex was entered as a first factor in a sequential sum of squares model. We tested for interaction effects by entering sex and the interactions of sex with the factors (1) stimulus category and (2) direction as additional fixed effects in the ANOVA models. In the left hemisphere, we observed a small, but significant, interaction effect between the factors sex and stimulus category $\left(\chi^{2}=7.88\right.$, $p=0.02)$. All other interaction effects with sex in both the left and the right hemisphere were not significant $(p>0.5)$. Based on these results, we further explored the effect of sex only within the left hemisphere. Using post hoc, two-sided paired $t$ tests between modulators of connection strength, we found a difference between the values of men and women with respect to the parameter for positive pictures that modulates the connection from the hippocampus to the amygdala (means: females BMA $=-7.62 \mathrm{e}-$ 04; males BMA $=0.0014 ; p=0.001$, Bonferroni-corrected for six tests). As the main and interaction effects of sex appeared rather small, we performed likelihood ratio tests between the models, which either (1) do include sex and the interactions of sex with the factors stimulus category and direction or (2) do not include any main or interaction effects of sex. Our analysis revealed that models containing sex and its interactions as factors are not sig- nificantly better $(p=0.07)$. We therefore used the more parsimonious ANOVA models, which did not include main or interaction effects of sex as additional factors, to analyze modulator values, as the variance explained by sex appeared to be minor.

Although the valence-dependent changes in connection strengths between the amygdala and the hippocampus were more pronounced in the left hemisphere, we refrained from exploring potential hemispheric differences, as a comparison of models and parameters in the context of DCM of fMRI data is only valid when all models are fitted to the same set of time courses (Friston et al., 2003).

In addition to modulators of connection strength, DCM also estimates intrinsic connectivity parameters. They represent the connectivity in the absence of experimental perturbations. The effective connection strength associated to an experimental condition can be calculated as the sum of the value of the intrinsic connection and the value of the modulator. We observed the following mean BMA intrinsic parameter estimates: connection from amygdala to hippocampus, 0.4188 (left hemisphere), 0.4643 (right hemisphere); connection from hippocampus to amygdala, 0.0355 (left hemisphere), 0.0173 (right hemisphere). The results for the intrinsic parameters suggest that the influence of the amygdala upon the hippocampus is $>10$ times stronger than the influence of the hippocampus upon the amygdala, even in the absence of experimental perturbations. A complete list of BMA parameter values for condition-specific modulators, intrinsic connections, and extrinsic inputs can be found in Tables 3 and 4 .

The enhanced memory performance for emotional pictures and the increased connection strength during the encoding of emotional pictures suggest that subsequently remembered pictures should be associated with increased connection strength, relative to subsequently not remembered pictures. Here we performed post hoc analyses to test this prediction explicitly. Based on the same time courses as described above, and our prior knowledge with regard to the site of input, we defined a model with two modulators on each connection: (1) a modulator for pictures that were subsequently remembered; and (2) a modulator for pictures subsequently not remembered. This model was used to reexplain our connectivity findings in terms of subsequent memory. It was applied separately in each hemisphere. Modulatory parameter estimates of each subject were entered into two-sided paired $t$ tests. The results showed that remembered pictures were indeed associated with increased connection strength, compared with the connection strength of not remembered pictures (Bonferroni multiple comparison corrected significance threshold for two separate hypothesis tests per hemisphere, $p=0.025)$. Connection from the amygdala to the hippocampus: left hemisphere remembered versus not remembered, $p=0.0024$; modulator remembered, $0.0776 \pm 0.0052$; modulator not remembered, $0.0572 \pm 0.0046$; right hemisphere remembered versus not remembered, $p=0.0025$; modulator remembered, $0.0940 \pm 0.0059$; modulator not remembered, $0.0723 \pm 0.0051$. Connection from the hippocampus to the amygdala: left hemisphere remembered versus not remembered, $p=1.3864 \mathrm{e}-08$; modulator remembered, $0.0026 \pm 0.0007$; modulator not remembered, $-0.0039 \pm 0.0007$; right hemisphere 
Table 2. Regions showing task-dependent activations related to the encoding of pictures (positive + negative + neutral - scrambled pictures), in addition to our Rol analysis $^{a}$

\begin{tabular}{|c|c|c|c|c|c|c|}
\hline \multirow[b]{2}{*}{ Cluster no. } & \multirow[b]{2}{*}{ Maximum $t$ value within cluster } & \multirow[b]{2}{*}{ Regional correspondence of the maximum } & \multicolumn{3}{|c|}{ MNI coordinates at maximum } & \multirow[b]{2}{*}{ No. of voxels } \\
\hline & & & $x$ & $y$ & $z$ & \\
\hline 1 & 62.17 & ctx-lh-superiorfrontal (4\%) & -8.25 & 55 & 28 & 549 \\
\hline 2 & 53.54 & ctx-lh-isthmuscingulate (94\%); ctx-lh-precuneus (6\%) & -2.75 & -49.5 & 24 & 142 \\
\hline 3 & 50.45 & ctx-Ih-parsorbitalis (10\%); ctx-ctx-lh-parstriangularis (3\%); Ih-lateralorbitofrontal (2\%) & -41.25 & 27.5 & -12 & 175 \\
\hline 4 & 49.24 & ctx-rh-lateraloccipital (23\%) & 38.5 & -82.5 & -8 & 144 \\
\hline 5 & 47.28 & right-cerebellum-cortex (99\%) & 24.75 & -79.75 & -36 & 23 \\
\hline 6 & 45.52 & ctx-lh-middletemporal (60\%); ctx-lh-superiortemporal (4\%) & -57.75 & -11 & -16 & 46 \\
\hline 7 & 45.09 & ctx-rh-middletemporal (70\%); ctx-rh-superiortemporal (4\%) & 55 & -5.5 & -20 & 47 \\
\hline 8 & 44.12 & ctx-Ih-inferiorparietal (65\%); ctx- ctx-Ih-supramarginal (3\%); Ih-middletemporal (2\%) & -49.5 & -63.25 & 24 & 34 \\
\hline 9 & 43.03 & ctx-rh-superiorparietal (42\%); ctx-rh-lateraloccipital (38\%); ctx-rh-cuneus (2\%) & 19.25 & -93.5 & 28 & 14 \\
\hline 10 & 42.75 & ctx-Ih-parahippocampal (99\%) & -19.25 & -35.75 & -16 & 8 \\
\hline 11 & 42.37 & left-cerebellum-cortex (96\%) & -24.75 & -79.75 & -36 & 8 \\
\hline 12 & 41.67 & ctx-rh-parahippocampal (99\%) & 22 & -33 & -16 & 3 \\
\hline 13 & 40.70 & ctx-Ih-lateraloccipital (24\%); ctx-lh-superiorparietal (17\%) & -13.75 & -93.5 & 24 & 10 \\
\hline 14 & 40.70 & ctx-rh-inferiorparietal (53\%); ctx-rh-middletemporal (7\%) & 55 & -60.5 & 16 & 22 \\
\hline 15 & 40.63 & ctx-rh-parstriangularis (55\%) & 52.25 & 27.5 & 0 & 7 \\
\hline 16 & 40.39 & ctx-rh-superiortemporal (78\%); ctx-rh-middletemporal (5\%) & 46.75 & 11 & -28 & 4 \\
\hline 17 & 40.33 & ctx-rh-isthmuscingulate $(5 \%)$ & 13.75 & -46.75 & 8 & 6 \\
\hline 18 & 40.09 & ctx-Ih-lateraloccipital (93\%); ctx-lh-inferiorparietal (3\%) & -46.75 & -82.5 & 4 & 3 \\
\hline 19 & 40.01 & ctx-rh-fusiform (94\%); ctx-rh-inferiortemporal (3\%) & 38.5 & -49.5 & -20 & 3 \\
\hline 20 & 39.81 & ctx-rh-superiorfrontal $(88 \%)$ & 8.25 & 16.5 & 64 & 2 \\
\hline
\end{tabular}

${ }^{a}$ Task-related associations across the whole brain. Because of the sample size and respective statistical power, we find large clusters of significant voxels throughout the brain (see Figure 5 ). The smallest $t$ value within our regions of interest served as the minimum threshold $(t=38.86, p<0.05$, whole-brain Bonferroni corrected) to highlight regions with comparable or larger task-related associations. Regions and probabilities are in accordance with the in-house atlas. ctx, Cortex; lh, left hemisphere; rh, right hemisphere.
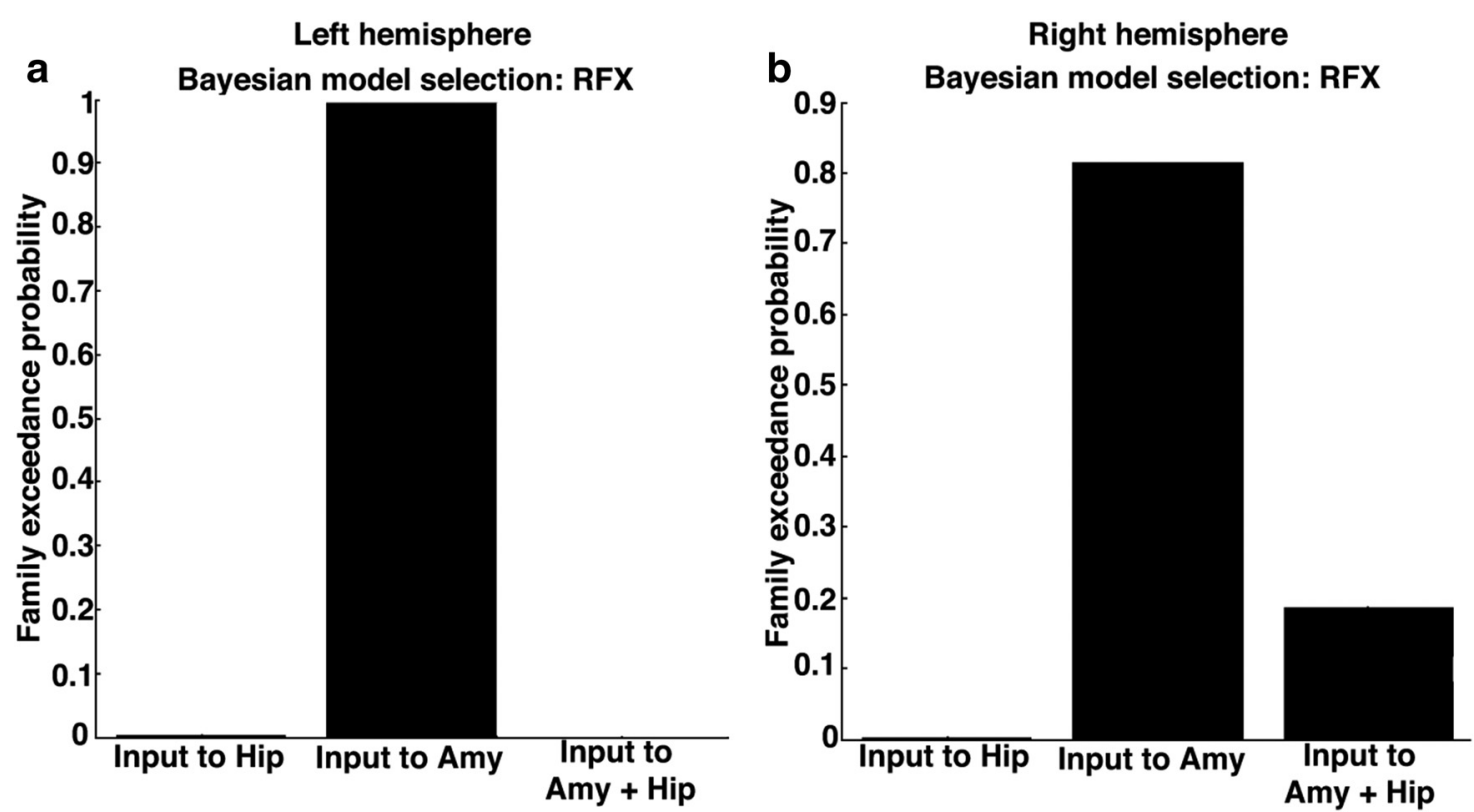

Figure 6. Model comparison: family exceedance probability. Comparison between the three model families, representing competing input possibilities to the network in (a) left hemisphere $(N=574)$ and $(\boldsymbol{b})$ right hemisphere $(N=573)$. Left hemisphere: (1) input to hippocampus (0.0002); (2) input to amygdala (0.9998); (3) input to both amygdala and hippocampus $(0)$. Right hemisphere: (1) input to hippocampus (0.0013); (2) input to amygdala (0.8146); (3) input to both amygdala and hippocampus (0.1841). Amygdala (Amy), Hippocampus (Hip).

remembered versus not remembered, $p=1.9457 \mathrm{e}-08$; modulator remembered, $0.0033 \pm 0.0006$; modulator not remembered, $-0.0029 \pm 0.0005$. Values are mean \pm SEM. Modulators did not differ significantly between females and males $(p>0.29)$.

Above, we reported that positive and negative pictures had a similar effect on the strength of the connection projecting from the amygdala to the hippocampus. Given the hypothesized relevance of this connection for both emotional and memory processes, we tested whether the effect of positive and negative pictures also depends on whether they are subsequently remembered or not. Based on the same time courses as described above, and our prior knowledge with regard to the site of input, we defined a model that uses separate modulators for subsequently remembered and subsequently not remembered pictures per stimulus category (i.e., six modulators in total per connection; Tables 5 and 6). The model was applied separately in each hemisphere. Within the connection from the amygdala to the hippocampus, the modulators for (1) positive remembered, (2) positive not remembered, (3) negative remembered, and (4) negative not remembered pictures were entered in ANOVA with stimulus type (levels: positive/negative) as one factor, memory (levels: remembered/not remembered) as the other factor, and 


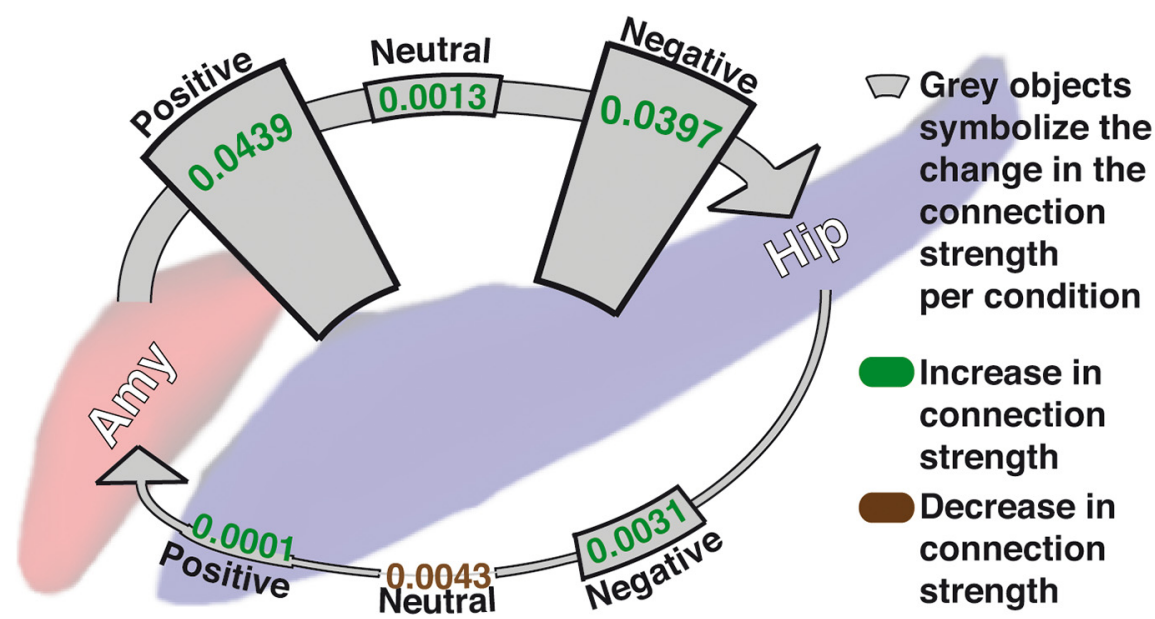

Figure 7. Change in connection strength between the amygdala (red) and the hippocampus (blue) during the encoding of positive, negative, and neutral pictures. Figure represents the results obtained in the left hemisphere. Top, Arrow indicates the influence that the amygdala exerts upon the hippocampus. Bottom, Arrow indicates the connection from the hippocampus to the amygdala. Values are model estimates, representing the change in connection strength per condition (mean of the BMA modulator values across all 574 subjects). See also Table 3 and Figure $8 a$; for the right hemisphere, see Figure $8 b$ and Table 4.

the interaction term between stimulus type and memory. Subjects were entered as random effect. Most of the variance was explained by the factor memory (left hemisphere, $\chi^{2}=17.28$, $p=3.22 \mathrm{e}-05$; right hemisphere, $\left.\chi^{2}=19.75, p=8.83 \mathrm{e}-06\right)$. The factor stimulus type explained less variance, and this effect did not reach significance in the right hemisphere (left hemisphere, $\chi^{2}=5.29, p=0.02$; right hemisphere, $\left.\chi^{2}=0.59, p=0.44\right)$. We observed that the effect of stimulus type did depend on memory (interaction between stimulus type and memory: left hemisphere, $\chi^{2}=8.27, p=0.004$; right hemisphere, $\chi^{2}=4.77, p=$ $0.03)$. We conducted two-sided paired post hoc $t$ tests between modulators to obtain more focused results (Bonferroni multiple comparison corrected significance threshold for two separate hypothesis tests per hemisphere: $p=0.025)$. In the case of remembered pictures, positive and negative stimuli had a similar effect on connection strength (left hemisphere, $p=0.60$; right hemisphere, $p=0.28$ ). In the case of subsequently not remembered pictures, negative stimuli had a stronger impact on connection strength than positive stimuli in the left hemisphere $(p=$ $0.0004)$, whereas their effect appeared to be similar in the right hemisphere $(p=0.06)$.

We used the contrast (positive + negative + neutral minus scrambled) to identify significantly activated voxels. As emotional conditions had twice the weight of the neutral condition, the extracted time courses may have been biased. We performed additional analyses without this bias by computing the effect of (1) positive and neutral and (2) negative and neutral pictures separately in two sets of distinct analyses. These analyses showed that our conclusions were not affected by this potential bias.

\section{Discussion}

By applying DCM of fMRI data, the present study revealed that the connection from the amygdala to the hippocampus (i.e., the influence that the amygdala exerts upon the hippocampus) is stronger during the encoding of emotional pictures than during the encoding of neutral pictures. Importantly, these findings apply both to the emotionally negative and positive valence. Our findings in humans therefore support the prediction of the memory-modulation hypothesis generated in animals, which states that the amygdala regulates information processing in the hip- pocampus. The valence- and directiondependent changes in connectivity were observed in both the left and right hemispheres. Within-subject analyses indicated that positive and negative pictures were rated as more arousing and were better remembered than neutral pictures (Figs. 3 and 4). However, between-subject analyses revealed that subjects who rated pictures as more arousing did not have a better memory performance than subjects who rated pictures as less arousing. Thus, the subjective arousal rating did not correlate with interindividual differences in memory performance.

The observation that positive and negative pictures have a similar effect on connection strength might be in line with earlier studies demonstrating that amygdala activation is associated with arousal common to both negative and positive events, but not valence itself (Anderson et al., 2003; Small et al., 2003; Kensinger and Corkin, 2004; Kensinger and Schacter, 2006; Lewis et al., 2007). However, although positive images were rated as less arousing than negative images in our study, they were slightly better remembered than negative pictures. This suggests that our behavioral and connectivity findings are not only a consequence of arousal but may also be influenced by additional factors, such as complexity, self-relevance, item familiarity, and semantic relatedness of the pictures (Kensinger, 2009). Nonetheless, differences with regard to arousal and memory were considerably smaller between positive and negative pictures than between emotional and neutral pictures (Figs. 3 and 4).

Previous studies in humans found enhanced episodic memory for emotional items already a few minutes after presentation (Hamann et al., 1999; de Quervain et al., 2007). Moreover, enhanced memory for emotional items has been found in experimental designs that alternate rapidly (within seconds) between emotional and neutral stimuli (Canli et al., 2000; de Quervain et al., 2007), suggesting that such memory-modulatory processes during encoding must be regulated in a quick and dynamic manner. Our present results indicate that the amygdala-hippocampal connectivity can quickly change (within seconds) in response to a rapid sequence of intermingled arousing and nonarousing stimuli and that the modulatory influence of the amygdala on the activity in the hippocampus is already present at the stage of stimulus presentation. This is in line with the idea that the amygdala alters the encoding of hippocampal-dependent, episodic memory by influencing perception and attention, such that emotional events receive priority (Hamann, 2001; Phelps, 2004; Todd et al., 2013). It remains to be investigated whether this influence during encoding serves as an "emotional tagging" to support a selective strengthening of emotionally arousing information during memory consolidation (Bergado et al., 2011). Although memory of emotional events can be enhanced without rehearsal (Harris and Pashler, 2005), the process of rehearsal has to be considered as a potential mechanism enhancing memory for emotionally arousing information. In the present study, the distracting working memory task between picture encoding and retrieval and the rapid succession of encoding events made rehearsal unlikely. 

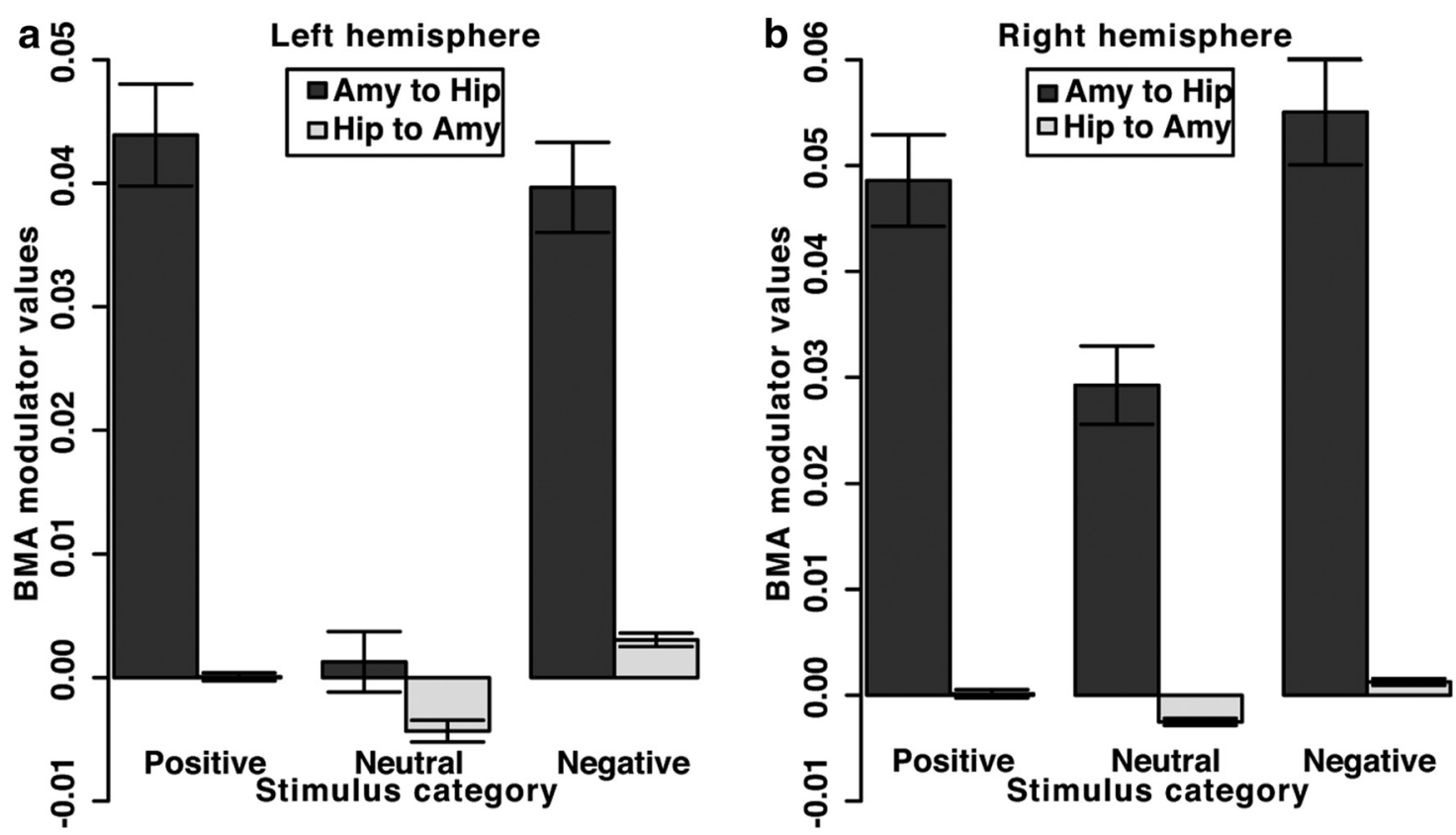

Figure 8. Plots illustrating the mean of the BMA modulator values per stimulus category and direction of connectivity across all subjects. Results obtained in $(\boldsymbol{a})$ left hemisphere $(N=574)$ and (b) right hemisphere $(N=573)$. Dark gray bars represent modulator values of the connection projecting from the amygdala to the hippocampus (Amy to Hip). Light gray bars represent modulator values of the connection that projects from the hippocampus to the amygdala (Hip to Amy). Error bars indicate the SEM. See also Tables 3 and 4.

Table 3. BMA parameter values obtained in the left hemisphere, related to results: parameter inference (Figs. 7 and 8$)^{a}$

\begin{tabular}{|c|c|c|c|c|c|c|}
\hline & \multicolumn{6}{|c|}{ Parameter values (left hemisphere) } \\
\hline Strength of driving input to amygdala & \multicolumn{6}{|l|}{$0.4868 \pm 0.0137$} \\
\hline Direction of connection & \multicolumn{3}{|c|}{ Amygdala to hippocampus } & \multicolumn{3}{|c|}{ Hippocampus to amygdala } \\
\hline \multirow[t]{2}{*}{ Strength of intrinsic connectivity } & $0.4188 \pm 0.0216$ & & & $0.0355 \pm 0.0073$ & & \\
\hline & Positive & Neutral & Negative & Positive & Neutral & Negative \\
\hline $\begin{array}{l}\text { Change in connection strength } \\
\text { (modulators) per condition }\end{array}$ & $0.0439 \pm 0.0041$ & $0.0013 \pm 0.0024$ & $0.0397 \pm 0.0036$ & $0.0001 \pm 0.0003$ & $-0.0043 \pm 0.0009$ & $0.0031 \pm 0.0005$ \\
\hline
\end{tabular}

${ }^{a}$ Values represent the mean \pm SEM of the BMA parameter values across all 574 subjects, based on models using one modulator per stimulus category and direction.

Table 4. BMA parameter values obtained in the right hemisphere, related to results: parameter inference (Fig. 8) ${ }^{a}$

\begin{tabular}{|c|c|c|c|c|c|c|}
\hline & \multicolumn{6}{|c|}{ Parameter values (right hemisphere) } \\
\hline Strength of driving input to amygdala & \multicolumn{6}{|l|}{$0.5263 \pm 0.0142$} \\
\hline Direction of connection & \multicolumn{3}{|c|}{ Amygdala to hippocampus } & \multicolumn{3}{|c|}{ Hippocampus to amygdala } \\
\hline \multirow[t]{2}{*}{ Strength of intrinsic connectivity } & $0.4643 \pm 0.0230$ & & & $0.0173 \pm 0.0033$ & & \\
\hline & Positive & Neutral & Negative & Positive & Neutral & Negative \\
\hline $\begin{array}{l}\text { Change in connection strength } \\
\text { (modulators) per condition }\end{array}$ & $0.0486 \pm 0.0043$ & $0.0293 \pm 0.0037$ & $0.0551 \pm 0.0050$ & $0.0001 \pm 0.0004$ & $-0.0025 \pm 0.0003$ & $0.0013 \pm 0.0003$ \\
\hline
\end{tabular}

${ }^{a}$ Values represent the mean \pm SEM of the BMA parameter values across all 573 subjects, based on models using one modulator per stimulus category and direction.

Furthermore, we reported that the modulation in connection strength from the amygdala to the hippocampus during the encoding of emotional pictures is substantially stronger than in the opposite direction. Nevertheless, we also observed a significant increase in connection strength from the hippocampus to the amygdala, although at a much smaller scale. This observation might be in line with the finding that hippocampal lesions prevent optimal amygdala activation during encoding of emotional material (Richardson et al., 2004). Previous studies demonstrate that the connectivity between the amygdala and the hippocampus varies depending on context. Processing differences in the retrieval of items associated with negative versus neutral contexts, independent of task requirements, were reflected in an increase in connection strength from the hippocampus to the amygdala (Smith et al., 2006). When retrieval of emotional information was relevant to current behavior, amygdala-hippocampal connectivity increased bidirectionally. A context-dependent influence of the hippocampus upon the amygdala has also been observed in animals (for review, see Maren et al., 2013). The observation that the hippocampus transmits contextual information to the amygdala might be in line with the idea that the brain uses an internal model of the environment when recognizing sensory input (Kiebel et al., 2009). Overall, these previous findings indicate that the current connectivity results may also depend on specific factors of the task. 
Table 5. Stimulus category and memory-specific modulator estimates obtained in the left hemisphere, related to results: parameter inference ${ }^{a}$

\begin{tabular}{|c|c|c|c|c|c|c|}
\hline \multirow[b]{3}{*}{ Direction of connection } & \multicolumn{6}{|c|}{ Parameter values (left hemisphere) } \\
\hline & \multicolumn{3}{|c|}{ Amygdala to hippocampus } & \multicolumn{3}{|c|}{ Hippocampus to amygdala } \\
\hline & Positive: rem & Neutral: rem & Negative: rem & Positive: rem & Neutral: rem & Negative: rem \\
\hline Change in connection strength (modulators) per condition & Positive: Not rem & Neutral: Not rem & Negative: Not rem & Positive: Not rem & Neutral: Not rem & Negative: Not rem \\
\hline
\end{tabular}

${ }^{a}$ Values represent the mean of parameter estimates across all 574 subjects, based on a model using one modulator per stimulus category, direction, and subsequent memory: rem, Remembered; Not rem, nonremembered.

Table 6. Stimulus category and memory-specific modulator estimates obtained in the right hemisphere, related to results: parameter inference ${ }^{a}$

\begin{tabular}{|c|c|c|c|c|c|c|}
\hline \multirow[b]{3}{*}{ Direction of connection } & \multicolumn{6}{|c|}{ Parameter values (right hemisphere) } \\
\hline & \multicolumn{3}{|c|}{ Amygdala to hippocampus } & \multicolumn{3}{|c|}{ Hippocampus to amygdala } \\
\hline & Positive: rem & Neutral: rem & Negative: rem & Positive: rem & Neutral: rem & Negative: rem \\
\hline \multirow[t]{3}{*}{ Change in connection strength (modulators) per condition } & $0.0453 \pm 0.0046$ & $0.0143 \pm 0.0033$ & $0.0386 \pm 0.0041$ & $0.0010 \pm 0.0003$ & $-0.0008 \pm 0.0003$ & $0.0035 \pm 0.0007$ \\
\hline & Positive: Not rem & Neutral: Not rem & Negative: Not rem & Positive: Not rem & Neutral: Not rem & Negative: Not rem \\
\hline & $0.0174 \pm 0.0039$ & $0.0265 \pm 0.0041$ & $0.0288 \pm 0.0040$ & $-0.0005 \pm 0.0005$ & $-0.0025 \pm 0.0004$ & $0.0011 \pm 0.0004$ \\
\hline
\end{tabular}

${ }^{a}$ Values represent the mean of parameter estimates across all 573 subjects, based on a model using one modulator per stimulus category, direction, and subsequent memory: rem, Remembered; Not rem, nonremembered.

In the present study, we focused on the connectivity between the amygdala and the hippocampus, as their involvement in the enhancement of memory for emotionally arousing events has been well established by animal experiments (Roozendaal and McGaugh, 2011). Activation of the BLA facilitates hippocampal LTP (Roozendaal and McGaugh, 2011), which has been related to synaptic plasticity and memory formation (Lynch, 2004). Moreover, activation of the BLA immediately after training facilitates memory consolidation by potentiating arousal-induced noradrenergic activation (McReynolds et al., 2010), which in turn increases hippocampal levels of activity-regulated cytoskeletal protein, an immediate-early gene implicated in hippocampal synaptic plasticity (McIntyre et al., 2005). Furthermore, lesions of the BLA or infusions of $\beta$-adrenoceptor antagonists into the BLA before training block the enhancement of memory consolidation induced by intrahippocampal, post-training infusions of glucocorticoid receptor agonists (Roozendaal and McGaugh, 1997; Roozendaal et al., 1999). Of note, the above-mentioned animal studies investigated the influence of the amygdala on the hippocampus exclusively in an emotionally aversive learning context.

Previous neuroimaging studies have indicated that the amygdala and the hippocampus are involved in the enhancing effect of emotional arousal on episodic memory encoding. A study in patients with variable degrees of left hippocampal and amygdala pathology indicates the importance of the intactness of these regions for optimal encoding of emotional material (Richardson et al., 2004). Furthermore, several studies in healthy humans have demonstrated coactivation of the amygdala and regions in the medial temporal lobe, such as the hippocampus, the entorhinal cortex, parahippocampal cortex, and perirhinal cortex (Hamann et al., 1999; Dolcos et al., 2004; Kensinger and Corkin, 2004; Ritchey et al., 2008). However, such correlational analyses are not informative with regard to the direction of a potential influence. In contrast, structural equation modeling (SEM) estimates the direction of the influence (i.e., effective connectivity) within a network and allows for a comparison of the connection strength between different conditions (Friston, 2011). By applying SEM on positron emission tomography data, it has been shown that the strength of the ipsilateral connections projecting from the right amygdala to the parahippocampal gyrus and from the right amygdala to the ventrolateral prefrontal cortex is increased during a negative relative to a neutral filmviewing condition (Kilpatrick and Cahill, 2003). A second study has investigated the effect of high and low arousing, subsequently remembered pictures within the negative and positive valence category by applying SEM on fMRI data. For negative information, arousal increased the strength of amygdala connections to the inferior frontal gyrus and the middle occipital gyrus, whereas for positive information arousal decreased the strength of these amygdala efferents (Mickley Steinmetz et al., 2010). These previous correlational or unidirectional SEM studies did not address the directionality of the influence (i.e., it has been unclear whether the amygdala primarily influences the hippocampus) or whether it is the hippocampus that predominantly influences the amygdala.

DCM infers connectivity by modeling neural dynamics with a system of differential equations. Therefore, the direction of influence and the condition-dependent modulation of connection strength can be more meaningfully determined than with static connectivity models, such as SEM (Penny et al., 2004; Friston, 2011). For a discussion on inference of causality in network estimation, see Smith et al. (2011). Another important advantage over SEM is that DCM strives for neurophysiological interpretability by making an explicit distinction between the "neural level" and the "hemodynamic level" (Penny et al., 2004). This is achieved by inverting a biophysically motivated and parameterized forward model, which links the modeled neural dynamics to the measured hemodynamic time courses (Friston et al., 2003). The connectivity parameters can therefore be interpreted as an influence between neural populations (Stephan et al., 2010).

Our inference on connectivity depends on the underlying mathematical assumptions incorporated in the parameterization of DCM. These assumptions have been critically assessed (Daunizeau et al., 2011; Roebroeck et al., 2011; Deshpande and Hu, 2012; Handwerker et al., 2012; Lohmann et al., 2012; Friston et al., 2013). For instance, the biophysical model for the interactions between neural states on one hand and the hemodynamic 
forward model on the other hand determines which of these models will absorb given aspects of the observed data (Roebroeck et al., 2011), and misestimation of the hemodynamic response may alter the predicted timing for neural events (Handwerker et al., 2012). In our current study, we report differences between modulators of connection strength. Such condition-dependent differences should be robust, even if the hemodynamic response was misspecified, as it is less likely to vary between experimental conditions than between brain regions or subjects (for a similar argument in the context of Granger Causality, see Roebroeck et al., 2005).

Two previous DCM studies examined connectivity during an associative emotional learning task. The first study demonstrated that frontal regions have a top-down influence on the amygdala, even though additional bottom-up connections from the amygdala to frontal regions are also likely part of the network (Curčić-Blake et al., 2012a). Moreover, the study showed, based on the most likely model, that top-down connections from frontal regions to the amygdala are increased during emotional conditions. The second study showed that the intrinsic connection from the inferior frontal gyrus to the parahippocampal gyrus varies between genotype groups (Curčić-Blake et al., 2012b). Our present analysis differs in terms of the network analyzed. Whereas the previous studies shed light on regions driving responses in the amygdala, we showed that the amygdala has a substantial downstream influence on activity in the hippocampus. Moreover, by modeling modulators of connection strength with respect to the positive, negative, and neutral condition, and by explicitly testing for differences between modulators, we were able to draw conclusions (1) with regard to similar or differential effects of these conditions on connection strength and (2) if conditions have a differential impact on connectivity depending on the direction of the influence.

Similar to most animal studies, our inference on connectivity is not necessarily restricted to direct anatomical connections between the amygdala and the hippocampus. Instead, the values of our connectivity parameters potentially reflect both direct and indirect connections between the regions. Therefore, even though emotionally positive and negative information may involve a different network consisting of several brain regions (Mickley Steinmetz et al., 2010), the net connectivity between the amygdala and hippocampus was similar for the two valences.

Together, the present DCM study provides evidence that, during encoding, emotionally arousing information leads to an increase in connectivity from the amygdala to the hippocampus regardless of its valence. As such, our results shed light on possible neural mechanisms underlying the enhanced episodic memory for emotional events in humans. Moreover, our findings may also provide guidance for future investigations of anxiety disorders, such as phobias and post-traumatic stress disorder, as they are characterized by both neural abnormalities in these regions and increased memory for emotionally aversive events (Coles and Heimberg, 2002; Shin et al., 2006; Etkin and Wager, 2007; Koenigs et al., 2008).

\section{References}

Anderson AK, Christoff K, Stappen I, Panitz D, Ghahremani DG, Glover G, Gabrieli JD, Sobel N (2003) Dissociated neural representations of intensity and valence in human olfaction. Nat Neurosci 6:196-202. CrossRef Medline

Ashburner J (2007) A fast diffeomorphic image registration algorithm. Neuroimage 38:95-113. CrossRef Medline

Bergado JA, Lucas M, Richter-Levin G (2011) Emotional tagging: a simple hypothesis in a complex reality. Prog Neurobiol 94:64-76. CrossRef Medline

Canli T, Zhao Z, Brewer J, Gabrieli JD, Cahill L (2000) Event-related activation in the human amygdala associates with later memory for individual emotional experience. J Neurosci 20:RC99. Medline

Coles ME, Heimberg RG (2002) Memory biases in the anxiety disorders. Clin Psychol Rev 22:587-627. CrossRef Medline

Curčić-Blake B, Swart M, Aleman A (2012a) Bidirectional information flow in frontoamygdalar circuits in humans: a dynamic causal modeling study of emotional associative learning. Cereb Cortex 22:436-445. CrossRef Medline

Curčić-Blake B, Swart M, Ter Horst G, Langers DR, Kema IP, Aleman A (2012b) Variation of the gene coding for DARPP-32 (PPP1R1B) and brain connectivity during associative emotional learning. Neuroimage 59:1540-1550. CrossRef Medline

Daunizeau J, David O, Stephan KE (2011) Dynamic causal modelling: a critical review of the biophysical and statistical foundations. Neuroimage 58:312-322. CrossRef Medline

de Quervain DJ, Kolassa IT, Ertl V, Onyut PL, Neuner F, Elbert T, Papassotiropoulos A (2007) A deletion variant of the $\alpha 2 \mathrm{~b}$-adrenoceptor is related to emotional memory in Europeans and Africans. Nat Neurosci 10:11371139. CrossRef Medline

Deshpande G, Hu X (2012) Investigating effective brain connectivity from fMRI data: past findings and current issues with reference to Granger causality analysis. Brain Connect 2:235-245. CrossRef Medline

Desikan RS, Ségonne F, Fischl B, Quinn BT, Dickerson BC, Blacker D, Buckner RL, Dale AM, Maguire RP, Hyman BT, Albert MS, Killiany RJ (2006) An automated labeling system for subdividing the human cerebral cortex on MRI scans into gyral based regions of interest. Neuroimage 31:968 980. CrossRef Medline

Dolcos F, LaBar KS, Cabeza R (2004) Interaction between the amygdala and the medial temporal lobe memory system predicts better memory for emotional events. Neuron 42:855-863. CrossRef Medline

Eichenbaum H (2004) Hippocampus: cognitive processes and neural representations that underlie declarative memory. Neuron 44:109-120. CrossRef Medline

Etkin A, Wager TD (2007) Functional neuroimaging of anxiety: a metaanalysis of emotional processing in PTSD, social anxiety disorder, and specific phobia. Am J Psychiatry 164:1476-1488. CrossRef Medline

Fischl B, Salat DH, Busa E, Albert M, Dieterich M, Haselgrove C, van der Kouwe A, Killiany R, Kennedy D, Klaveness S, Montillo A, Makris N, Rosen B, Dale AM (2002) Whole brain segmentation: automated labeling of neuroanatomical structures in the human brain. Neuron 33:341355. CrossRef Medline

Friston KJ (2011) Functional and effective connectivity: a review. Brain Connect 1:13-36. CrossRef Medline

Friston KJ, Harrison L, Penny W (2003) Dynamic causal modelling. Neuroimage 19:1273-1302. CrossRef Medline

Friston K, Daunizeau J, Stephan KE (2013) Model selection and gobbledygook: response to Lohmann et al. Neuroimage 75:275-278; discussion 279-281. CrossRef Medline

Hamann S (2001) Cognitive and neural mechanisms of emotional memory. Trends Cogn Sci 5:394-400. CrossRef Medline

Hamann SB, Ely TD, Grafton ST, Kilts CD (1999) Amygdala activity related to enhanced memory for pleasant and aversive stimuli. Nat Neurosci 2:289-293. CrossRef Medline

Handwerker DA, Gonzalez-Castillo J, D’Esposito M, Bandettini PA (2012) The continuing challenge of understanding and modeling hemodynamic variation in fMRI. Neuroimage 62:1017-1023. CrossRef Medline

Harris CR, Pashler H (2005) Enhanced memory for negatively emotionally charged pictures without selective rumination. Emotion 5:191-199. CrossRef Medline

Kensinger EA (2009) What factors need to be considered to understand emotional memories? Emot Rev 1:120-121. CrossRef Medline

Kensinger EA, Corkin S (2004) Two routes to emotional memory: distinct neural processes for valence and arousal. Proc Natl Acad Sci U S A 101: 3310-3315. CrossRef Medline

Kensinger EA, Schacter DL (2006) Processing emotional pictures and words: effects of valence and arousal. Cogn Affect Behav Neurosci 6:110 126. CrossRef Medline

Kiebel SJ, Klöppel S, Weiskopf N, Friston KJ (2007) Dynamic causal mod- 
eling: a generative model of slice timing in fMRI. Neuroimage 34:14871496. CrossRef Medline

Kiebel SJ, von Kriegstein K, Daunizeau J, Friston KJ (2009) Recognizing sequences of sequences. PLoS Comput Biol 5:e1000464. CrossRef Medline

Kilpatrick L, Cahill L (2003) Amygdala modulation of parahippocampal and frontal regions during emotionally influenced memory storage. Neuroimage 20:2091-2099. CrossRef Medline

Klein A, Andersson J, Ardekani BA, Ashburner J, Avants B, Chiang MC, Christensen GE, Collins DL, Gee J, Hellier P, Song JH, Jenkinson M, Lepage C, Rueckert D, Thompson P, Vercauteren T, Woods RP, Mann JJ, Parsey RV (2009) Evaluation of 14 nonlinear deformation algorithms applied to human brain MRI registration. Neuroimage 46:786-802. CrossRef Medline

Koenigs M, Huey ED, Raymont V, Cheon B, Solomon J, Wassermann EM, Grafman J (2008) Focal brain damage protects against post-traumatic stress disorder in combat veterans. Nat Neurosci 11:232-237. CrossRef Medline

Lang P, Bradley M, Cuthbert B (2008) International affective picture system (IAPS): affective ratings of pictures and instruction manual. Technical Report A-8. Gainesville, FL: University of Florida.

Leff AP, Schofield TM, Stephan KE, Crinion JT, Friston KJ, Price CJ (2008) The cortical dynamics of intelligible speech. J Neurosci 28:13209-13215. CrossRef Medline

Lewis PA, Critchley HD, Rotshtein P, Dolan RJ (2007) Neural correlates of processing valence and arousal in affective words. Cereb Cortex 17:742748. CrossRef Medline

Lohmann G, Erfurth K, Müller K, Turner R (2012) Critical comments on dynamic causal modelling. Neuroimage 59:2322-2329. CrossRef Medline

Lynch MA (2004) Long-term potentiation and memory. Physiol Rev 84:87136. CrossRef Medline

Maren S, Phan KL, Liberzon I (2013) The contextual brain: implications for fear conditioning, extinction and psychopathology. Nat Rev Neurosci 14:417-428. CrossRef Medline

Marreiros AC, Kiebel SJ, Friston KJ (2008) Dynamic causal modelling for fMRI: a two-state model. Neuroimage 39:269-278. CrossRef Medline

McGaugh JL (2002) Memory consolidation and the amygdala: a systems perspective. Trends Neurosci 25:456-461. CrossRef Medline

McGaugh JL (2003) Memory and emotion: the making of lasting memories (maps of the mind). New York: Columbia UP.

McIntyre CK, Miyashita T, Setlow B, Marjon KD, Steward O, Guzowski JF, McGaugh JL (2005) Memory-influencing intra-basolateral amygdala drug infusions modulate expression of Arc protein in the hippocampus. Proc Natl Acad Sci U S A 102:10718-10723. CrossRef Medline

McReynolds JR, Donowho K, Abdi A, McGaugh JL, Roozendaal B, McIntyre CK (2010) Memory-enhancing corticosterone treatment increases amygdala norepinephrine and Arc protein expression in hippocampal synaptic fractions. Neurobiol Learn Mem 93:312-321. CrossRef Medline

Mickley Steinmetz KR, Addis DR, Kensinger EA (2010) The effect of arousal on the emotional memory network depends on valence. Neuroimage 53:318-324. CrossRef Medline

Nieuwenhuys R, Voogd J, Huijzen C (2008) The human central nervous system, Ed 4. Berlin: Springer.

Penny WD, Stephan KE, Mechelli A, Friston KJ (2004) Modelling functional integration: a comparison of structural equation and dynamic causal models. Neuroimage 23 [Suppl 1]:S264-S274.

Penny WD, Stephan KE, Daunizeau J, Rosa MJ, Friston KJ, Schofield TM, Leff AP (2010) Comparing families of dynamic causal models. PLoS Comput Biol 6:e1000709. CrossRef Medline
Phelps EA (2004) Human emotion and memory: interactions of the amygdala and hippocampal complex. Curr Opin Neurobiol 14:198-202. CrossRef Medline

R Core Team (2012) R: a Language and Environment for Statistical Computing. Vienna: R Foundation for Statistical Computing.

Richardson FM, Seghier ML, Leff AP, Thomas MS, Price CJ (2011) Multiple routes from occipital to temporal cortices during reading. J Neurosci 31:8239-8247. CrossRef Medline

Richardson MP, Strange BA, Dolan RJ (2004) Encoding of emotional memories depends on amygdala and hippocampus and their interactions. Nat Neurosci 7:278-285. CrossRef Medline

Ritchey M, Dolcos F, Cabeza R (2008) Role of amygdala connectivity in the persistence of emotional memories over time: an event-related FMRI investigation. Cereb Cortex 18:2494-2504. CrossRef Medline

Roebroeck A, Formisano E, Goebel R (2005) Mapping directed influence over the brain using Granger causality and fMRI. Neuroimage 25:230 242. CrossRef Medline

Roebroeck A, Formisano E, Goebel R (2011) The identification of interacting networks in the brain using fMRI: model selection, causality and deconvolution. Neuroimage 58:296-302. CrossRef Medline

Roozendaal B, McGaugh JL (1997) Basolateral amygdala lesions block the memory-enhancing effect of glucocorticoid administration in the dorsal hippocampus of rats. Eur J Neurosci 9:76-83. CrossRef Medline

Roozendaal B, McGaugh JL (2011) Memory modulation. Behav Neurosci 125:797-824. CrossRef Medline

Roozendaal B, Nguyen BT, Power AE, McGaugh JL (1999) Basolateral amygdala noradrenergic influence enables enhancement of memory consolidation induced by hippocampal glucocorticoid receptor activation. Proc Natl Acad Sci U S A 96:11642-11647. CrossRef Medline

Saunders RC, Rosene DL, Van Hoesen GW (1988) Comparison of the efferents of the amygdala and the hippocampal formation in the rhesus monkey: II. Reciprocal and non-reciprocal connections. J Comp Neurol 271: 185-207. CrossRef Medline

Seghier ML, Price CJ (2010) Reading aloud boosts connectivity through the putamen. Cereb Cortex 20:570-582. CrossRef Medline

Shin LM, Rauch SL, Pitman RK (2006) Amygdala, medial prefrontal cortex, and hippocampal function in PTSD. Ann N Y Acad Sci 1071:67-79. CrossRef Medline

Sladky R, Friston KJ, Tröstl J, Cunnington R, Moser E, Windischberger C (2011) Slice-timing effects and their correction in functional MRI. Neuroimage 58:588-594. CrossRef Medline

Small DM, Gregory MD, Mak YE, Gitelman D, Mesulam MM, Parrish T (2003) Dissociation of neural representation of intensity and affective valuation in human gustation. Neuron 39:701-711. CrossRef Medline

Smith AP, Stephan KE, Rugg MD, Dolan RJ (2006) Task and content modulate amygdala-hippocampal connectivity in emotional retrieval. Neuron 49:631-638. CrossRef Medline

Smith SM, Miller KL, Salimi-Khorshidi G, Webster M, Beckmann CF, Nichols TE, Ramsey JD, Woolrich MW (2011) Network modelling methods for FMRI. Neuroimage 54:875-891. CrossRef Medline

Stephan KE, Penny WD, Daunizeau J, Moran RJ, Friston KJ (2009) Bayesian model selection for group studies. Neuroimage 46:1004-1017. CrossRef Medline

Stephan KE, Penny WD, Moran RJ, den Ouden HE, Daunizeau J, Friston KJ (2010) Ten simple rules for dynamic causal modeling. Neuroimage 49: 3099-3109. CrossRef Medline

Todd RM, Schmitz TW, Susskind J, Anderson AK (2013) Shared neural substrates of emotionally enhanced perceptual and mnemonic vividness. Front Behav Neurosci 7:40. CrossRef Medline 